\title{
Multinodular and Vacuolating Neuronal Tumor of the Cerebrum: A New "Leave Me Alone" Lesion with a Characteristic Imaging Pattern
}

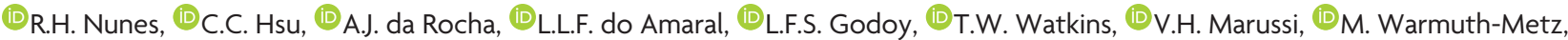

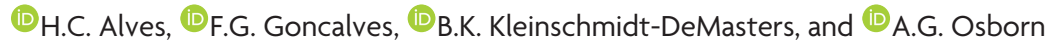

\begin{abstract}
SUMMARY: Multinodular and vacuolating neuronal tumor of the cerebrum is a recently reported benign, mixed glial neuronal lesion that is included in the 2016 updated World Health Organization classification of brain neoplasms as a unique cytoarchitectural pattern of gangliocytoma. We report 33 cases of presumed multinodular and vacuolating neuronal tumor of the cerebrum that exhibit a remarkably similar pattern of imaging findings consisting of a subcortical cluster of nodular lesions located on the inner surface of an otherwise normal-appearing cortex, principally within the deep cortical ribbon and superficial subcortical white matter, which is hyperintense on FLAIR. Only 4 of our cases are biopsy-proven because most were asymptomatic and incidentally discovered. The remaining were followed for a minimum of 24 months (mean, 3 years) without interval change. We demonstrate that these are benign, nonaggressive lesions that do not require biopsy in asymptomatic patients and behave more like a malformative process than a true neoplasm.
\end{abstract}

ABBREVIATIONS: DNET = dysembryoplastic neuroepithelial tumor; MVNT = multinodular and vacuolating neuronal tumor of the cerebrum

M ultinodular and vacuolating neuronal tumor of the cerebrum (MVNT) was first described in 2013 as a benign seizure-associated lesion with a characteristic histopathologic appearance. ${ }^{1}$

The most recent (2016) World Health Organization Classification of Tumors of the Central Nervous System ${ }^{2}$ includes MVNT as a unique cytoarchitectural pattern of gangliocytoma, though to date, it remains unclear whether MVNT is a true neoplastic process or a dysplastic hamartomatous/malformative lesion. ${ }^{2,3}$

Only a few cases of MVNT have been reported in the neuropathology literature. ${ }^{1,3-6}$ We report 33 cases of presumed MVNT that exhibit a remarkably similar pattern of imaging findings that,

Received February 18, 2017; accepted after revision May 3.

From the Division of Neuroradiology (R.H.N., A.J.d.R., L.L.F.d.A., H.C.A.), Santa Casa de Sao Paulo School of Medical Sciences, Sao Paulo, Brazil; Division of Neuroradiology (R.H.N., A.J.d.R.), Diagnosticos da America SA, Sao Paulo, Brazil; Department of Medical Imaging (C.C.H., T.W.W.), Princess Alexandra Hospital, Brisbane,

Queensland, Australia; Division of Neuroradiology (L.L.F.d.A., V.H.M.), Medimagem Hospital da Beneficencia Portuguesa de Sao Paulo, Sao Paulo, Brazil; Department of Radiology (L.F.S.G.), Hospital Sirio Libanes, Sao Paulo, Brazil; Department of Radiology (L.F.S.G.), Medical School, Universidade de Sao Paulo, Sao Paulo, Brazil; Department of Neuroradiology (M.W.-M), University Hospital Würzburg, Würzburg, Germany; Department of Radiology (F.G.G.), Children's Hospital of Brasilia, Brasilia, Brazil; Department of Pathology (B.K.K.-D.), University of Colorado, Aurora, Colorado; and Department of Radiology (A.G.O.), University of Utah, Salt Lake City, Utah.

Please address correspondence to Renato Hoffmann Nunes, MD, Santa Casa de Misericórdia de São Paulo, Serviço de Diagnóstico por Imagem, Rua Dr Cesário Motta Junior 112, Vila Buarque, São Paulo-SP/Brazil, 01221-020; e-mail: renatohn@hotmail.com; @renatohn

三 Indicates article with supplemental on-line table.

http://dx.doi.org/10.3174/ajnr.A5281 in many of our cases, were followed for years without demonstrating interval change. Only 4 of our cases were biopsy-proven because most were asymptomatic and incidentally discovered.

In this article, we describe the nearly pathognomonic imaging appearance of MVNTs and suggest that these are nonaggressive "leave me alone" brain lesions, which do not require either biopsy or resection.

\section{CASE SERIES}

\section{Case Selection}

Fifty-three cases of presumed MVNTs were collected from the MR imaging archives of the multinational contributing authors. Imaging was performed between July 2006 and November 2016. All cases were characterized as a subcortical or juxtacortical lesion consisting of a small cluster of nodules (Fig 1). Patient records for each case were retrospectively reviewed, and demographic features, clinical symptoms prompting initial imaging, histopathology reports, and follow-up studies or subsequent development of neurologic symptoms were noted by each contributing author. Cases that did not have either biopsy proof or follow-up of at least 24 months were later excluded, resulting in 33 patients.

\section{Imaging Analysis}

All imaging studies were performed on either a $1.5 \mathrm{~T}$ or a $3 \mathrm{~T}$ scanner. In all studies, sequences performed included sagittal T1WI, axial FLAIR, and DWI. Depending on the scanner used, either a gradient-echo sequence or a susceptibility-weighted imaging sequence was performed. Thin-section steady-state sequences (FIESTA, 

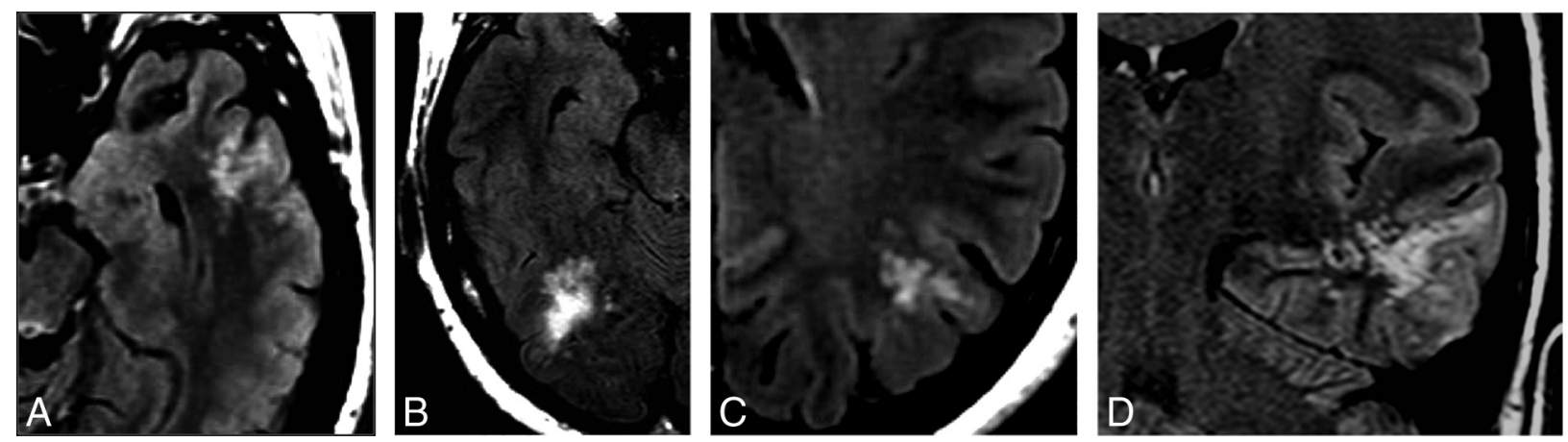

FIG 1. Typical imaging presentation on FLAIR. Selected axial $(A-C)$ and coronal $(D)$ FLAIR images of presumed MVNT demonstrate supratentorial hyperintense intra-axial lesions consisting of groups of multiple nodules, located on the subcortical ribbon and superficial subcortical white matter following the gyral contour. The images illustrate the variability in the size and location of the lesions and the degree of surrounding signal change (A, Case 21. B, Case 23. C, Case 7. D, Case 19).

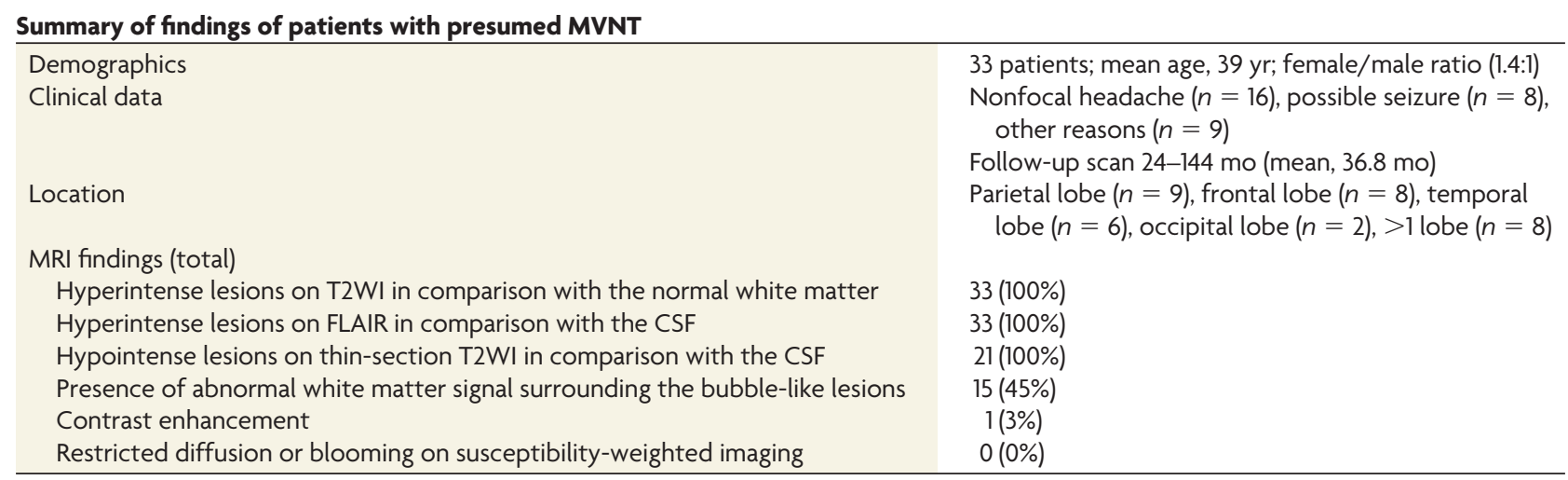

balanced FFE, CISS) were occasionally acquired. Gadolinium-enhanced T1WI was performed in all patients. Three neuroradiologists (R.H.N., A.J.d.R., and A.G.O., with 5, 22, and $>35$ years of experience, respectively) reviewed all individual cross-sectional images in a consensus analysis. Location, configuration, lesion size, and signal intensity on T1WI and T2WI, FLAIR, DWI, thin-section steady-state sequences, and postgadolinium T1WI were tabulated.

\section{RESULTS}

\section{Demographic Data}

Patient demographics, clinical presentation, and imaging findings in all 33 patients are summarized in the Table and detailed in the On-line Table. Patient ages ranged from 8 to 63 years, with a mean of 39 years (median, 41 years) at the time of initial diagnosis. There were 19 females and 14 males (female/male ratio, 1.4:1).

\section{Imaging Findings}

Size and Configuration. All lesions consisted of groupings of multiple $(3$ to $>10$ ) tiny discrete, sharply marginated, round or ovoid intra-axial nodules, ranging from 1 to $5 \mathrm{~mm}$ in diameter. They were all supratentorial and located on the inner surface of an otherwise normal-appearing cortex. The lesions often surrounded a sulcus, principally within the deep cortical ribbon and superficial subcortical white matter (Figs 1-3). Overall lesion size ranged from to 7 to $57 \mathrm{~mm}$ in maximum diameter, and mass effect was minimal or absent.

Location. Nine of $33(27 \%)$ lesions were located in the parietal lobe, 8 (24\%) were confined to the frontal lobe, and 6 (18\%) were isolated to the temporal lobe. Only 2 lesions were located in the occipital lobe (4\%). Eight (24\%) affected more than 1 lobe.

Signal Intensity. Twenty-nine nodules (88\%) were mildly hypo-/ isointense relative to normal white matter, while the remainder were hypointense on T1-weighted spin-echo sequences. All nodules did not suppress on FLAIR (Figs 2-4) and were hyperintense on T2WI to normal white matter and hypointense in comparison with CSF. The differences in signal intensity on T2WI were better demonstrated on thin-section steady-state sequences (Fig 2), which were available for review in 21 patients $(64 \%)$. The white matter surrounding or adjacent to the small nodules appeared normal in nearly half of patients (18/33). In $45 \%$, confluent T2/FLAIR hyperintensity that spared the overlying cortex was present (Figs 1-3). No lesions exhibited restricted diffusion (Fig 3), and none showed blooming on susceptibility-weighted imaging.

Contrast Enhancement. Post-contrast enhancement was absent in all (Fig 3) except 1 lesion (97\%). This lesion displayed a very subtle focus of enhancement along its medial margin.

\section{Clinical Data}

Presenting symptoms included nonfocal headache $(n=16)$ and suspected seizure $(n=8)$. Nine patients were scanned for other reasons, such as Bell's palsy, glioma, meningioma follow-up, neuroimaging surveillance of patients with known skin melanoma, and psychiatric evaluations.

Follow-up scans were available in all except 3 cases (all 
of which were biopsy-proven), ranging from 24 to 144 months (mean, 36.8 months) following initial imaging. All $(100 \%)$ were clinically indolent and unchanged in size or appearance.
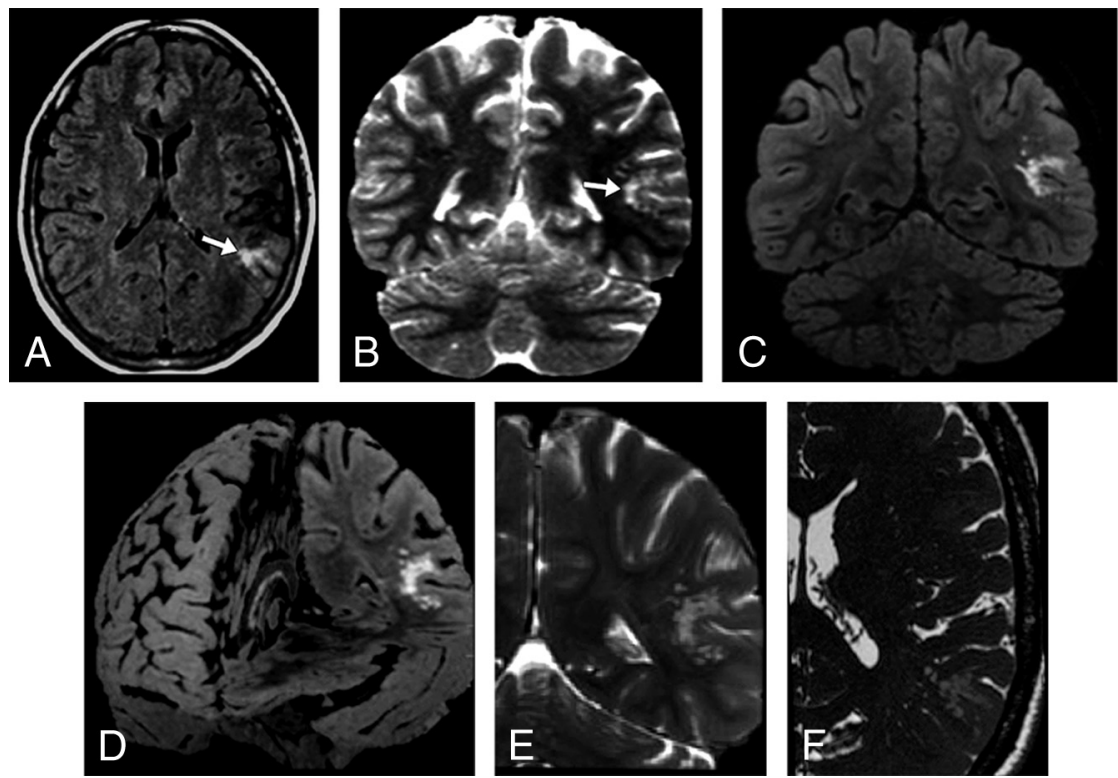

FIG 2. A 47-year-old woman who presented with nonfocal headache (case 1). Axial FLAIR (A) and coronal T2-weighted I (B) MR images demonstrate a left temporoparietal hyperintense subcortical lesion (arrows). The lesion had ill-defined borders, and the surrounding white matter appeared slightly hyperintense on T2/FLAIR. After 60 months of clinical stability, the study was performed on a 3T scanner. Coronal FLAIR $(C)$, FLAIR 3D reconstruction $(D)$, selected coronal T2WI $(E)$, and selected axial thin-section T2WI $(F)$ show the unchanged lesion located on the inner surface of otherwise normal-appearing left temporoparietal cortex surrounding a sulcus. The groups of multiple coalescent intralesional and perilesional nodules are better depicted. The images illustrate that the signal changes previously demonstrated in the surrounding white matter consist of innumerable very tiny nodules clustered together, sparing the overlying cortex.

\section{Pathologic Findings}

Because of their benign appearance and lack of relationship to specific clinical symptomatology, in most cases, the lesions were thought to be incidental. However, in 4 patients, the presenting clinical symptoms were potentially attributed to the identified lesion.

In 1 (case 10), even though the presenting symptoms (psychiatric) did not correlate with the lesion location, the neurosurgeons decided to resect the lesion to exclude a worrisome pathologic condition. Although initially the pathologic analysis was called "inconclusive," subsequent re-analysis of the slides showed multiple glioneuronal hamartomas fitting the histopathologic description of MVNT.

Another patient (case 26) presented with isolated seizure localized to the right temporal region, which correlated with the location of the subcortical lesion. The lesion was resected and the patient's symptoms resolved. Again, the initial pathologic analysis was called "inconclusive"; however, re-analysis of the histopathology showed 2 small areas of abnormal tissue consisting of glial and neuronal cells in a rarified background of focal microcyst formation, consistent with the histopathologic description of MVNT.

In another (case 31), the patient
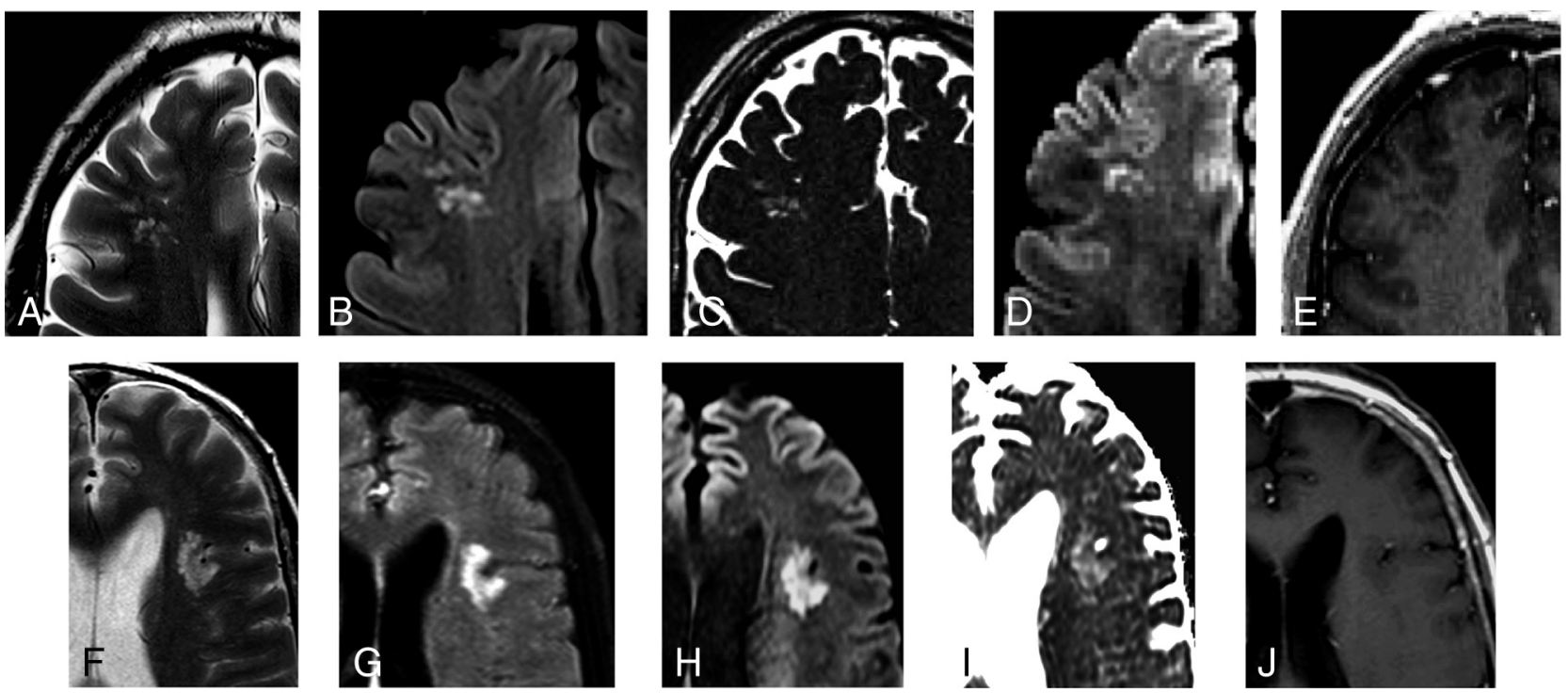

FIG 3. Most prevalent MR imaging presentations. A 50-year-old asymptomatic man with a history of skin melanoma (case 15). A-E, Selected axial MR images show a right frontal subcortical lesion consisting of groups of multiple nodules, hyperintense on FLAIR/T2WI ( $A$ and $B$ ). Selected axial thin-section T2WI (C) depicts mild hypointensity in comparison with the CSF. High signal is seen on an axial DWI (D) with high ADC values (not shown) due to the T2-shinethrough effect. No abnormal enhancement is depicted (E). No changes were detected on imaging follow-up after 25 months (not shown). A 41-year-old man who presented with nonfocal headache (case 8). F-J, Selected axial MR images reveal a left opercular subcortical lesion consisting of groups of multiple nodules, hyperintense on FLAIR/T2WI $(F$ and $G)$. High signal is seen on an axial DWI $(H)$ with high ADC values (I) due to the T2-shinethrough effect. No abnormal enhancement is depicted ( $)$. No changes were detected on imaging follow-up after 24 months (not shown). 

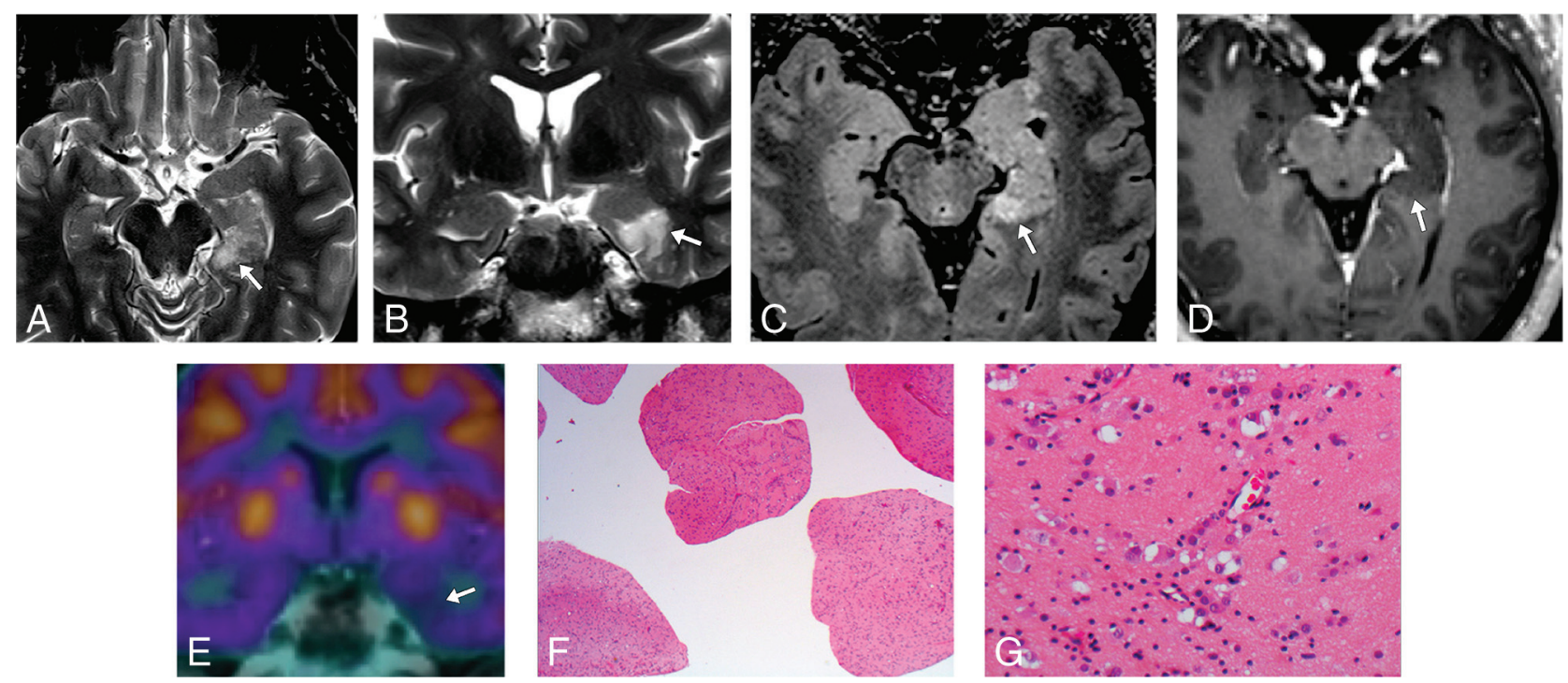

FIG 4. A 53-year-old male patient with temporal lobe epilepsy (case 31). A-D, MR imaging demonstrates a probable MVNT in the left parahippocampal gyrus (arrows). E, FDG-PET MR brain imaging shows focal hypometabolism in the left medial temporal lobe corresponding to the site of suspected lesion. $F$ and $G$, Hematoxylin-eosin-stained histopathology slides demonstrate the abnormal clustering and vacuolation of the neuronal cells. Images were obtained with $20 \times$ magnification $(F)$ and $200 \times$ magnification $(G)$.
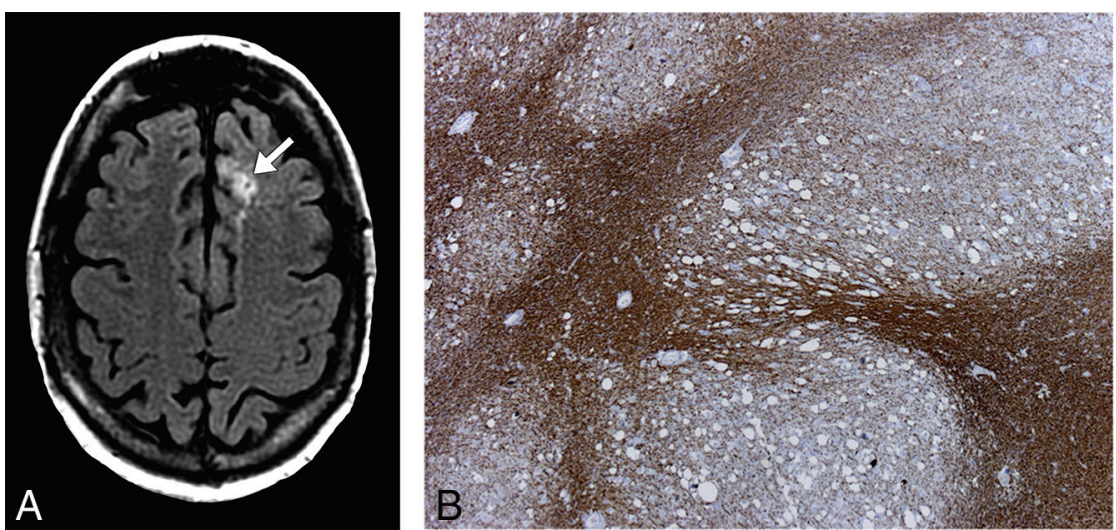

FIG 5. A 43-year-old male patient with headaches (case 33). A, Axial FLAIR image demonstrates a left frontal lobe lesion (arrow) consisting of multiple coalescent T2 and FLAIR hyperintense nodules located in the deep cortical ribbon and without mass effect. B, Low-power photomicrograph with immunohistochemistry staining for neurofibrillary protein shows positive staining between variably sized nonstaining vacuolating nodules.

presented with temporal lobe epilepsy. MRI evaluation showed a lesion in the left parahippocampal gyrus (Fig 4). Surgical resection of the lesion was performed and histopathology analysis revealed abnormal brain tissue in the left parahippocampal gyrus that included a population of cells showing neuronal features, many of which had vacuolation of a perikarya and a degree of clustering. Immunohistochemical stains confirmed a degree of nodularity and a weak immunoreactivity for synaptophysin on many neuronal cells, but most appeared negative for neuronal nuclear protein and neurofilament protein. There was variable background of positive dendritic staining for CD34. The lesion was also negative for IDH1-R132H mutation antibody, and staining was normal for ATRX and $p 53$. The Ki67 proliferation refraction was very low, and staining for GFAP showed varying background gliosis. Findings supported the diagnosis of MVNT.

In the other pathology-proven case (case 33), the patient pre- sented with headaches (Fig 5). Brain MR imaging was performed and demonstrated a left frontal lobe lesion suggestive of MVNT. Histopathology and immunohistochemical stains confirmed the diagnosis.

\section{DISCUSSION}

MVNTs are distinctive neuronal lesions first described by Huse et al ${ }^{1}$ in 2013 in a small case series of 10 patients. Since then, an additional 5 cases have been reported in the neuropathology literature. $^{3-6}$ Because so few reported cases were available, the new 2016 World Health Organization Classification of CNS neoplasms recognized MVNT as an entity but provisionally included it as a unique cytoarchitectural pattern of gangliocytoma, noting that it was unclear whether it is a truly neoplastic or dysplastic lesion. ${ }^{2}$ This article examines the imaging and clinical spectrum of a large series of presumed MVNTs and delineates classic imaging findings of MVNT in an effort to prevent misdiagnosis and unnecessary biopsy.

Published cases ${ }^{1,3-6}$ share similar histopathologic features, showing neuroepithelial cells with conspicuous stromal vacuolation arranged in nodules principally within the deep cortical ribbon and superficial subcortical white matter. These dysplastic cells typically orient perpendicular to the cortical surface, which correlates well with the radiologic appearance of clusters or a linear array of juxtacortical nodules. The constituent dysplastic cells exhibit immunopositivity for $\mathrm{HuC} / \mathrm{HuD}$ and Olig2, associated with neurogenesis at an earlier stage of neuronal development. However, they show negativity or weak positivity for neuronal lineage markers that are present in mature neurons, therefore, revealing an early neuronal immunophenotype. To date, genetic 


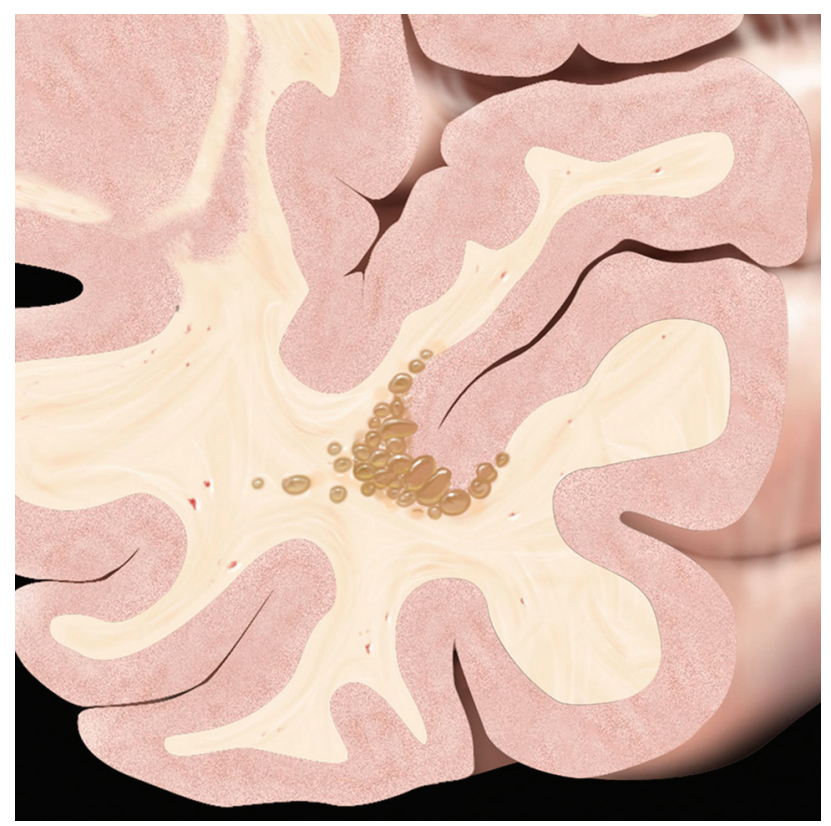

FIG 6. MVNT typical presentation graphic illustration. Schematic coronal view depicts a nodular "bubbly-appearing" lesion located on the inner surface of otherwise normal-appearing left temporal lobe cortex within the deep cortical ribbon and superficial subcortical white matter. Reprinted from Osborn's Brain ${ }^{9}$ with permission from Elsevier.

analyses have failed to demonstrate characteristic molecular changes that specify a particular tumor subtype. ${ }^{1,3-6}$ More recently, MVNTs have been shown to demonstrate overexpression of $\alpha$-internexin, which is a major component of neurofilaments in interneurons and cerebellar granule cells. INA expression is seen in developing immature neurons. Again, these findings support the hypothesis that MVNT may represent a dysplastic early neuronal phenotype and may fall into a category between cortical malformation and harmatomatous lesions, which behave in a benign fashion., 3

MVNTs exhibit a highly characteristic appearance on MR imaging. All lesions in our series consist of a cluster of variably sized nodular lesions located on the subcortical ribbon and superficial subcortical white matter following the gyral contour (Fig 6). They appear hyperintense on T2WI, do not suppress on FLAIR, and typically do not enhance or cause mass effect (Figs 2-4). Our findings are in accordance with those in the cases in the pathology literature. ${ }^{1,3-6}$ The nonsuppression on FLAIR and the slightly hypointense signal compared with CSF on T2-weighted sequences observed in this series are possibly related to a high protein or solid component within the vacuolated areas. In almost half of our patients, the subcortical white matter adjacent to the small nodules are hyperintense on T2/FLAIR; this appearance is likely due to coalescence of many smaller clustered nodules and partial volume averaging (Fig 2), sometimes resembling a mass-like lesion as previously described. ${ }^{3-6}$

Most of our cases were imaged before the description of MVNT in the 2016 World Health Organization Classification and were initially diagnosed as enlarged perivascular spaces, focal cortical dysplasia, or dysembryoplastic neuroepithelial tumor (DNET). Although MVNT may have some overlapping imaging features with DNET and other cortically based tumors, the absence of cortical involvement and prominent mass effect and intra- or perilesional tumor nodules help to distinguish MVNTs. ${ }^{3,6,7}$ Enlarged perivascular spaces may also have some similar imaging features; however, they typically exhibit CSF-like signal on all sequences, while MVNTs do not suppress on FLAIR. Prominent perivascular spaces that are below the resolution of 1.5 or 3T MR imaging may not suppress on FLAIR, but this pattern is usually described as an abnormal white matter signal surrounding other typical enlarged perivascular spaces, particularly in the anterior temporal region. ${ }^{8}$

While the most common clinical manifestation was an episode of seizure in the neuropathology literature,,$^{1,3-6}$ in this series, possible seizure was only observed in a few patients (19\%) and the remainder presented with nonspecific, nonfocal clinical manifestations. Most MVNTs are usually an incidental finding on imaging studies and may be more common than other neuronal and mixed glioneuronal tumors. The true prevalence of this lesion in asymptomatic individuals may have been underestimated in the neuropathology literature due to reporting bias because most are probably not resected.

It is still not clear whether multinodular and vacuolating neuronal tumor represents a neoplasm or malformation ${ }^{2}$; however, on the basis of our results, given the long stability of the imaging findings and clinical course, it is apparent that MVNT behaves in a benign, nonaggressive manner that is more like a hamartomatous lesion than a neoplasm. Imaging findings of MVNT are highly suggestive of the diagnosis. While a few MVNTs may be causative of seizure disorders and therefore require excision for clinical management, akin to DNET, most are asymptomatic lesions that are "leave me alone" entities. These lesions usually do not require biopsy, resection, or follow-up imaging unless and until it can be demonstrated that the visualized lesion is responsible for the symptoms.

Our retrospective study had clear limitations. Only half of our patients had adequate imaging follow-up to confirm stability with time. Another major limitation is that histopathology was only available for 4 cases. Nevertheless, the imaging findings of all presumed MVNTs were strikingly similar to the ones described in the neuropathology literature.

\section{CONCLUSIONS}

This large case series describes the typical MRI appearance of MVNT characterized by subcortical cluster of FLAIR hyperintense nodular lesions located on the inner surface of an otherwise normal-appearing cortex without enhancement or significant mass effect. Although the true nature of MVNT remains to be fully elucidated, the histopathology literature to-date favors a malformative lesion over a neoplasm. We emphasize that MVNT is a "leave me alone" brain lesion. When incidentally discovered, MVNT can be sufficiently managed on surveillance imaging to ensure stability and unnecessary surgery or biopsy should be avoided.

\section{ACKNOWLEDGMENTS}

We thank Thomas Robertson, BMedSc, MBBS(Hons), FRCPA, from the Department of Anatomical Pathology and Cytopathology, Royal Brisbane and Women's Hospital, Herston, Queens- 
land, Australia, and Vaska Kasturi, FRCPA, from the Department of Pathology, Princess Alexandra Hospital, Australia, for their significant contribution in the pathology evaluation.

Disclosures: Ann G. Osborn—UNRELATED: Consultancy: Elsevier, Comments: led the Brain Team for neuroradiology content for books, STATdx, RADPrimer, 2014 to present; Payment for Lectures Including Service on Speakers Bureaus: Mallinckrodt, Comments: Neuroradiology from the Masters course, 2014-2015; Royalties: Elsevier, Comments: book royalties; Stock/Stock Options: Amirsys, Comments: exercised in June 2014 during acquisition of Amirsys from Elsevier.

\section{REFERENCES}

1. Huse JT, Edgar M, Halliday J, et al. Multinodular and vacuolating neuronal tumors of the cerebrum: 10 cases of a distinctive seizureassociated lesion. Brain Pathol 2013;23:515-24 CrossRef Medline

2. Louis DN, Perry A, Reifenberger G, et al. The 2016 World Health Organization Classification of Tumors of the Central Nervous System: a summary. Acta Neuropathol 2016;131:803-20 CrossRef Medline

3. Yamaguchi M, Komori T, Nakata Y, et al. Multinodular and vacuolat- ing neuronal tumor affecting amygdala and hippocampus: a quasitumor? Pathol Int 2016;66:34-41 CrossRef Medline

4. Bodi I, Curran O, Selway R, et al. Two cases of multinodular and vacuolating neuronal tumour. Acta Neuropathol Commun 2014;2:7 CrossRef Medline

5. Fukushima S, Yoshida A, Narita Y, et al. Multinodular and vacuolating neuronal tumor of the cerebrum. Brain Tumor Pathol 2015;32: 131-36 CrossRef Medline

6. Nagaishi M, Yokoo H, Nobusawa S, et al. Localized overexpression of alpha-internexin within nodules in multinodular and vacuolating neuronal tumors. Neuropathology 2015;35:561-68 CrossRef Medline

7. Shin JH, Lee HK, Khang SK, et al. Neuronal tumors of the central nervous system: radiologic findings and pathologic correlation. $\mathrm{Ra}$ diographics 2002;22:1177-89 CrossRef Medline

8. Rawal S, Croul SE, Willinsky RA, et al. Subcortical cystic lesions within the anterior superior temporal gyrus: a newly recognized characteristic location for dilated perivascular spaces. AJNR Am J Neuroradiol 2014;35:317-22 CrossRef Medline

9. Osborn AG. Osborn's Brain. 2nd ed. Philadelphia: Elsevier; 2017:592 